\title{
Emergence of anomalous transport in stressed rough fractures
}

\author{
Peter K. Kang ${ }^{\mathrm{a}, \mathrm{b}}$, Stephen Brown ${ }^{\mathrm{c}}$, Ruben Juanes ${ }^{\mathrm{a}, \mathrm{c}, *}$ \\ ${ }^{a}$ Dept. of Civil and Environmental Engineering, Massachusetts Institute of Technology, \\ 77 Massachusetts Avenue, Cambridge, Massachusetts, USA \\ ${ }^{b}$ Center for Water Resource Cycle Research, Korea Institute of Science and Technology, \\ Seoul, South Korea \\ ${ }^{c}$ Dept. of Earth, Atmospheric and Planetary Sciences, Massachusetts Institute of \\ Technology, 77 Massachusetts Avenue, Cambridge, Massachusetts, USA
}

\begin{abstract}
We report the emergence of anomalous (non-Fickian) transport through a roughwalled fracture as a result of increasing normal stress on the fracture. We show that the origin of this anomalous transport behavior can be traced to the emergence of a heterogeneous flow field dominated by preferential channels and stagnation zones, as a result of the larger number of contacts in a highly stressed fracture. We show that the velocity distribution determines the late-time scaling of particle spreading, and velocity correlation determines the magnitude of spreading and the transition time from the initial ballistic regime to the asymptotic anomalous behavior. We also propose a spatial Markov model that reproduces the transport behavior at the scale of the entire fracture with only three physical parameters. Our results point to a heretofore unrecognized link between geomechanics and particle transport in fractured media.
\end{abstract}

Keywords: Groundwater flow, anomalous transport, fracture, roughness, spatial Markov model

\footnotetext{
* Corresponding author

Email address: juanes@mit.edu (Ruben Juanes)
} 


\section{Introduction}

Fluid flow and tracer transport through geologic fractures play a critical role in many subsurface processes, including groundwater contamination and remediation, nuclear waste disposal, hydrocarbon recovery, geothermal energy extrac-

5 tion, hydraulic fracturing, and induced seismicity (Bear et al., 1993 , Moreno \& Neretnieks, 1993; Bodvarsson et al., 1999: Pruess, 2006, Yasuhara et al., 2006). It has been shown - with theoretical and numerical models, as well as with laboratory and field experiments - that macroscopic transport through fracture networks is often anomalous (Berkowitz \& Scher, 1997, Geiger et al., 2010

10 Kang et al. 2011b a, 2015b), characterized by heavy-tailed particle distribution density, both in space and time, and nonlinear temporal evolution of particle mean square displacement (MSD) (Shlesinger, 1974, Bouchaud \& Georges, 1990 Metzler \& Klafter, 2000).

It is well known that matrix diffusion can induce anomalous transport (Car15 rera et al. 1998), but our interest here is in rock formations like fractured granite, where the role of matrix diffusion is relatively minor and can often be neglected (Becker \& Shapiro, 2000). Geologic fractures, however, are always under significant overburden stress. While confining stress has been shown to impact fluid flow through rough-walled fractures in a fundamental way (e.g., Unger

20 \& Mase, 1993, Olsson \& Brown, 1993, Pyrak-Nolte \& Morris, 2000, Watanabe et al., 2008: Auradou, 2009, Nemoto et al., 2009: Watanabe et al., 2013; Ishibashi et al., 2015: Pyrak-Nolte \& Nolte, 2016), studies of anomalous transport at the scale of individual fractures have so far either ignored the potential role of confining stress (Måløy et al., 1988; Detwiler et al., 2000; Auradou et al., 2001

25 Bodin et al. 2003a; Drazer et al., 2004, Talon et al., 2012, Wang \& Cardenas, 2014), relied on nonmechanistic models (Tsang \& Tsang, 1987), or focused on the role of shear stress (Koyama et al. 2008, Vilarrasa et al., 2011; Jing et al. 2013). As a result, the mechanistic underpinning and theoretical modeling for the emergence of anomalous transport in rough fractures under normal stress remains unexplored. 
Here, we demonstrate that an increase in the normal stress on a rough fracture can induce anomalous transport. Normal stress transforms the fracture geometry from a relatively homogeneous to a very heterogeneous flow structure: as the mean fracture aperture decreases, the flow organizes into preferential-flow channels and stagnation zones. To study the impact of normal stress on flow and transport, we first generate rough fracture surfaces, solve the nonlocal elastic contact problem on the rough-walled fractures under normal stress, and solve the flow and transport problem through the stressed rough-walled fractures. Then, by quantitatively analyzing the key mechanisms that lead to anomalous transport, we develop a parsimonious model of the transport dynamics - the proposed model can reproduce the transport behavior at the scale of the entire fracture with only three physical parameters. Our findings advance our understanding of transport through fractured media by linking anomalous transport behavior with the geomechanical properties of fractures and their state of stress.

\section{2. Generating rough fracture surfaces}

Geologic fractures typically exhibit a complex surface topography [Figure[1(a)]. Here, we construct realistic rough fracture surfaces using the spectral synthesis method, which captures the fundamental property that fractures are self-affine fractal surfaces (Power \& Tullis, 1991). The methodology relies on two key ingredients: a power spectral density function and a phase spectrum (Power \& Tullis, 1991; Brown, 1995, Glover et al., 1998). The power spectral density of real fracture surfaces exhibits power-law decay as a function of the wavenumber $k$ (inverse of wavelength $\lambda$ ), where the exponent is determined by the fractal dimension $D_{f}$ of the fracture surface, and the intercept is determined by the standard deviation of surface heights, $\sigma_{f}$. The phase spectrum, in contrast, is a nearly random process, independent of frequency (white noise).

Experimental observations also indicate that the top and bottom fracture surfaces are strongly correlated for long wavelengths but poorly correlated for short wavelengths (Brown, 1995 , Glover et al., 1998). This is physically intu- 
(a)

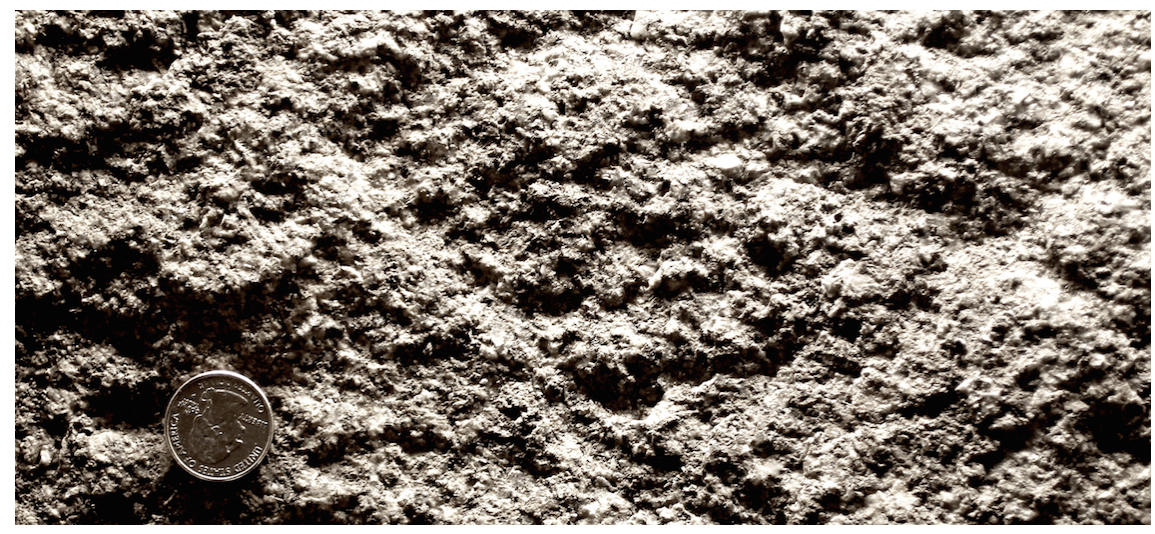

(b)

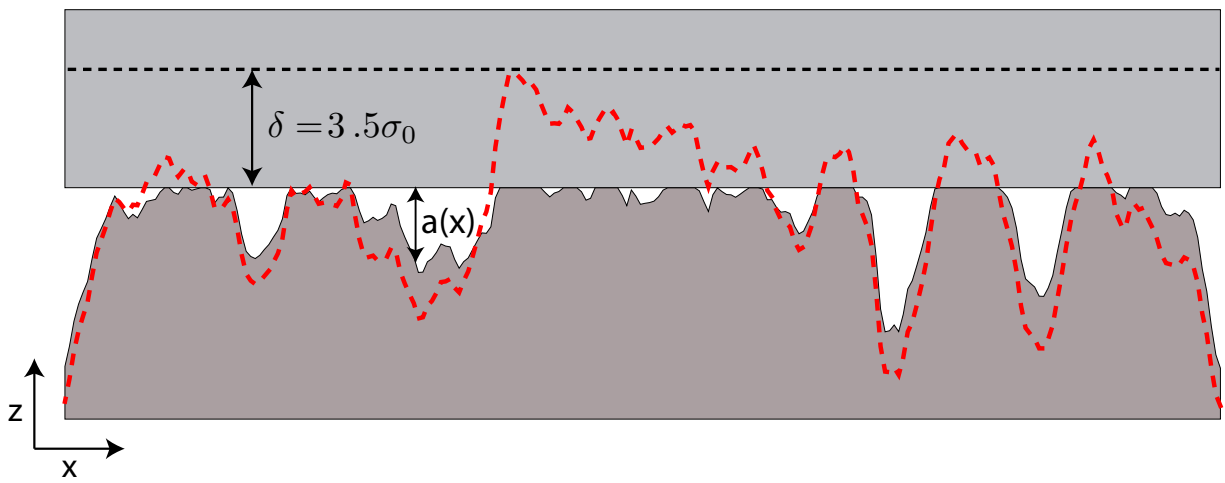

Figure 1: (a) Grayscale photograph of a rough fracture surface of granite, obtained from an outcrop in Vermont, USA. Self-affine fractal surfaces are known to be good representations of natural fractures. (b) As the normal displacement $\delta$ increases, contact areas appear; we solve the nonlocal elastic contact problem to obtain aperture fields. Aperture profiles at $\delta=0$ and at $\delta=3.5 \sigma_{0}$ are shown. $\delta=0$ corresponds to the state when the two surfaces are at first contact (zero normal stress). 
itive: top and bottom surfaces that were once perfectly mated will maintain similar large-scale structures, but small-scale structures will be disturbed during fracturing process. To incorporate this fundamental observation into the synthetic rough surface generator, and to allow for a gradual decay in correlation with wavenumber, we introduce the phase correlation function $\gamma=$

${ }_{65} \frac{1}{2}\left[1+\operatorname{erf}\left(-\left(k-k_{c}\right) / \theta\right)\right]$, where $\theta$ is a model parameter that determines the rate of correlation decay, and $k_{c}=1 / \lambda_{c}$ is the wavenumber at where the phase correlation between the top and bottom surface is 0.5 .

Once the power and phase spectra of the top and bottom surfaces have been set, we generate the two surfaces by performing an inverse Fourier transform. The aperture field at first contact $\left(a_{\delta=0}(x, y)\right)$ can be obtained by substracting top and bottom surfaces heights such that the minimum aperture is zero without deformation; $\delta$ is the compressive normal displacement from first contact [Figure 1(b)]. For the aperture field presented in this study, the mean aperture value at first contact is $\bar{a}_{\delta=0}=4.3 \sigma_{0}$ where $\sigma_{0}$ is the standard deviation of aperture values at $\delta=0$. For the results presented in this paper, we choose $D_{f}=2.3, \theta=4, \lambda_{c}=L / 15, \sigma_{f}=L / 100$ where $L$ is the domain size, and digital resolution of $256 \times 256$ pixels.

\section{Elastic contact problem}

We obtain the geometry of the stressed rough fracture by solving an elastic contact problem on the synthetic rough surface subject to normal stress [Figure 1(b)]. Fractured rock often exhibits elastic behavior, and past studies employed an elastic model to investigate the role of normal stress on rough surfaces (e.g., Bandis et al. 1983; Brown \& Scholz, 1985, Hopkins, 1990; Unger \& Mase, 1993; Pyrak-Nolte \& Morris, 2000, Petrovitch et al., 2014). We employ 2000, Pollard \& Fletcher, 2005, Johnson \& DeGraff, 1988): shear modulus $G=20 \mathrm{GPa}$, and Poisson ratio $\nu=0.25$, which are in the range of common चalues for natural rocks such as granite, basalt, limestone and sandstone (John- 
son \& DeGraff, 1988). We consider the long-range deformation produced by the

force at each contact, and the combined effect of multiple contacts Andrews 1988, Unger \& Mase, 1993). As the distance between the top and bottom surfaces decreases due to an increase in confining stress, a region of interpenetration between the top and bottom surfaces emerges. We constrain the deformation such that there is no interpenetration between the two surfaces. This is a mixed boundary value problem with displacement prescribed over part of the surface and normal stress prescribed over the remainder of the surface. The analytical solution for vertical/normal displacement due to a point force on an elastic half space is known as the Boussinesq solution, $B(r)=\frac{(1-\nu)}{2 \pi G} \frac{1}{r}$, where $r$ is the distance from the point force. The normal displacement $w(x, y)$ is obtained by convolution of the Boussinesq solution with the stress field $S(x, y)$ : $w(x, y)=\iint S\left(x^{\prime}, y^{\prime}\right) B(r) d x^{\prime} d y^{\prime}$ Andrews, 1988; Unger \& Mase, 1993). We use the discrete Fourier transform to solve the mixed boundary value problem, which makes the solution periodic.

By solving the elastic deformation problems for increasing values of the normal displacement $\delta$, we obtain aperture maps at different levels of stress. The probability distributions of aperture values at four different displacements $\left(\delta=0,1.5 \sigma_{0}, 2.5 \sigma_{0}\right.$, and $\left.3.5 \sigma_{0}\right)$ clearly show a dramatic change in the aperture heterogeneity [Figure 2(d)]. The aperture distribution is initially Gaussian but becomes heavy tailed as contact areas emerge. A broad aperture distribution implies a broad local (pixel-sale) fracture conductivity, which in turn impacts flow and transport. We note that solving the elastic deformation problem leads to different fracture geometry compared with simply removing the overlaps between the two surfaces, especially at high normal stress.

\section{Impact of stress on the flow field}

To study the impact of stress on the flow field, we perform a fluid flow simulation for incompressible fluid with constant viscosity and density on the final solution of the elastic deformation simulation at each value of the displacement 
(a)

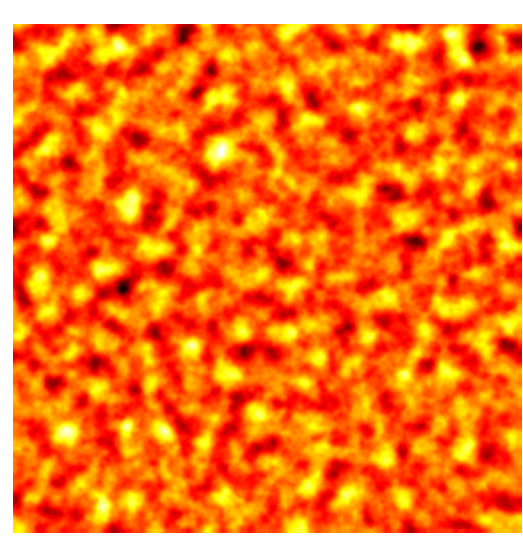

(d) 10

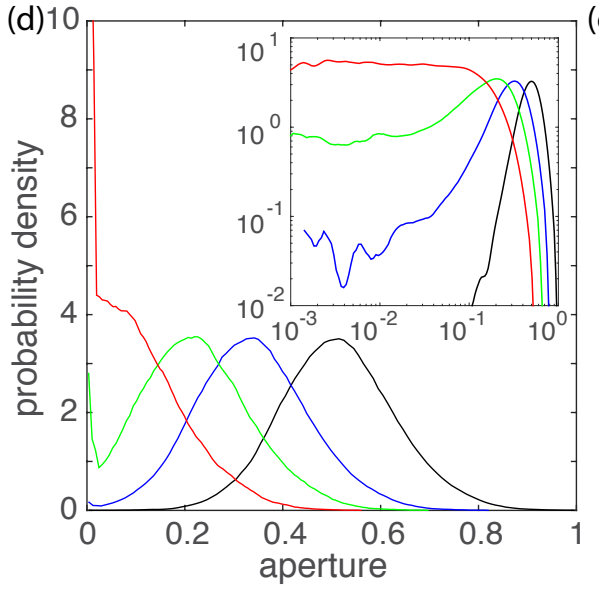

(b)

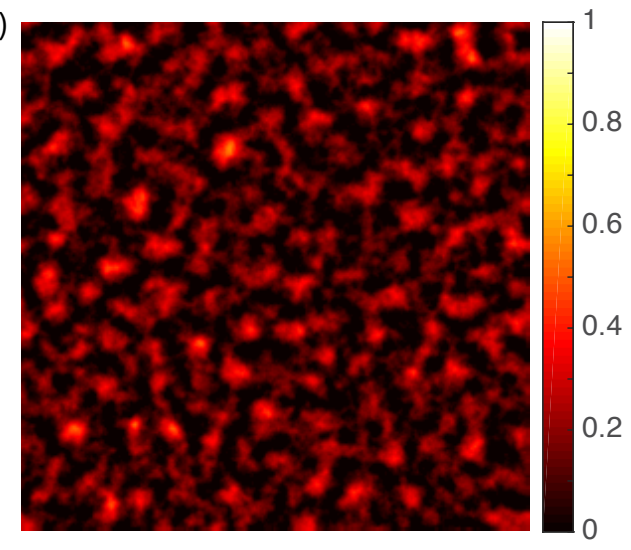

(c)

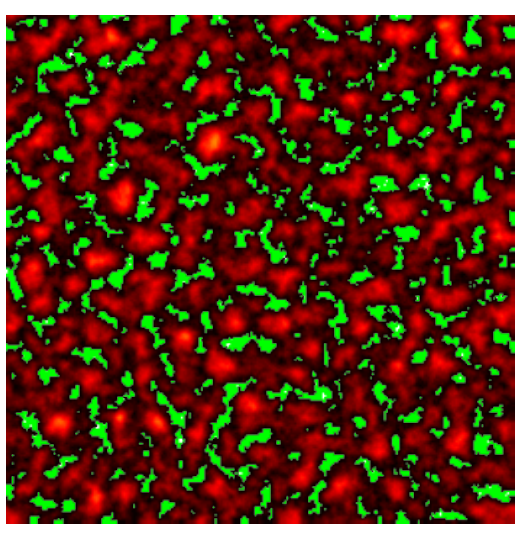

Figure 2: (a) Aperture map $a(x, y)$ between the two rough fracture surfaces under zero normal stress, when top and bottom surfaces are at first contact, $\delta=0$. Aperture values are normalized with the maximum aperture at $\delta=0$. (b) Aperture map $a(x, y)$ at displacement $\delta=3.5 \sigma_{0}$. Aperture values are normalized with the maximum aperture at $\delta=0$. (c) Same as (b) but the zero-aperture regions marked with green color to highlight the spatial organization of contact areas. (d) Probability density distributions of aperture at four different displacements: $\delta=0$ (black), $1.5 \sigma_{0}$ (blue), $2.5 \sigma_{0}$ (green), and $3.5 \sigma_{0}$ (red). As stress increases, the aperture distribution becomes broader and strongly skewed. Inset: the same plot in log-log scale. 
$\delta$ [Figure 1(b)]. We take the aperture map, $a(x, y)$, as the gap width in an equivalent parallel plate model (Moreno et al., 1988). By applying the lubrication approximation, we obtain a Darcy type equation for the gap-averaged fluid velocity, $\mathbf{u}=-\frac{a^{2}}{12 \eta} \nabla P$, where $\eta$ is the fluid dynamic viscosity and $P$ is the fluid pressure (Tsang \& Tsang, 1987, Moreno et al., 1988; Koyama et al. 2008: Watanabe et al., 2013: Ishibashi et al., 2015). The local cubic law is in general applicable when the flow is laminar and the aperture variation is small compared to the size of the grid block (Bodin et al., 2003b). When the aperture variation becomes comparable to the grid block size, 3D effects should be taken into account. Conservation of mass imposes that the gap-integrated velocity, or volumetric flux, $\mathbf{q}=\mathbf{u} a$, be divergence free: $\nabla \cdot \mathbf{q}=0$. We obtain the fluid pressure field in the $2 \mathrm{D}$ domain, $P(x, y)$, by solving the continuity equation with no-flow boundary conditions at the top and bottom boundaries, and fixed pressure values at the left (inlet, $P_{\text {in }}=1$ ) and right (outlet, $P_{\text {out }}=0$ ) boundaries.

The flow field through the fracture exhibits a fundamental change as a function of normal stress (Figure 3). Preferential flow paths with fast velocities [Figure 3(b)], stagnation zones with small velocities, and back-flow zones with negative velocities [Figure 3(c)] all emerge as stress increases. We quantify the change in the flow field by plotting the Eulerian velocity distribution at different stress states [Figure 3(d)]. The Eulerian velocity distribution has a well defined mean and variance at small stress values but converges towards a truncated power-law distribution as stress increases, indicating the dramatic impact of normal stress on flow.

\section{Emergence of anomalous transport}

Once the steady-state fluxes are obtained, we simulate transport of a passive tracer by particle tracking. We assume complete mixing at the computational grid blocks (Kang et al. 2011b, Moreno \& Neretnieks, 1993). Thus, the direction which the particle exits a grid block is chosen randomly with flux-weighted 
(a)
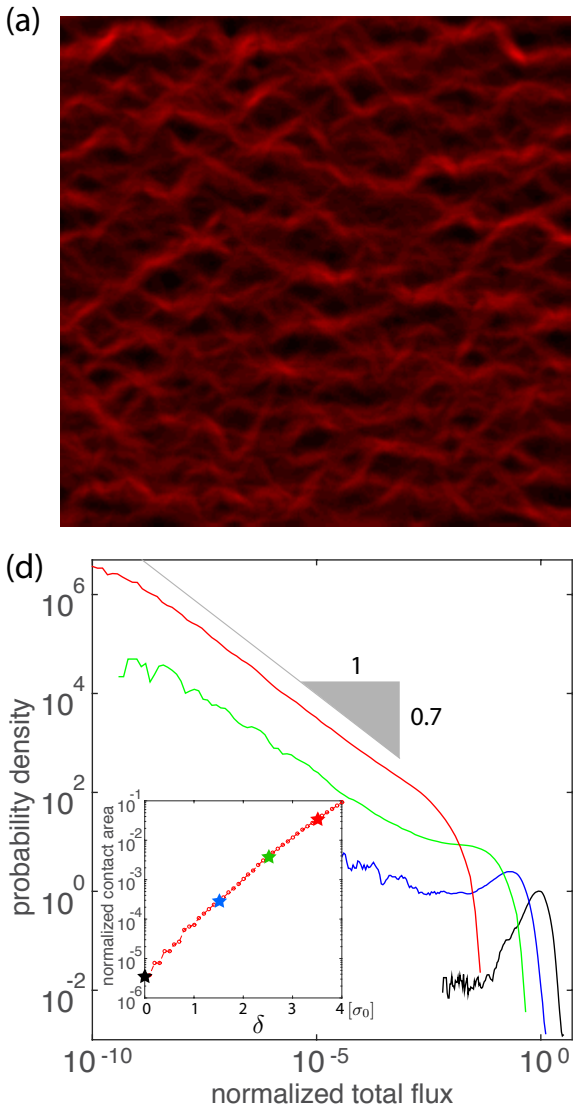

(b)

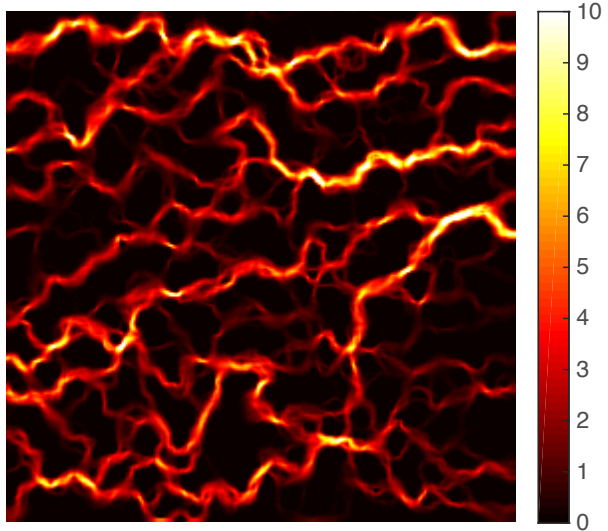

(c)

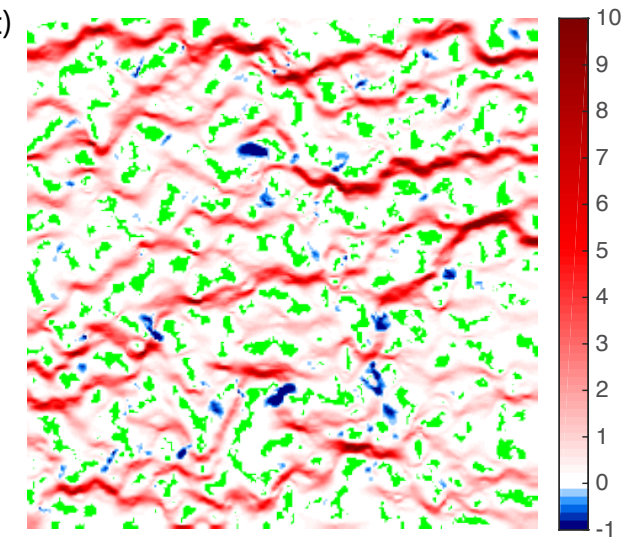

Figure 3: (a) Magnitude of the volumetric flux, $\frac{1}{2} \sum_{j}\left|q_{i j}\right|$, at each discretization grid block obtained from the solution of the flow equation on the rough fracture at first contact, $\delta=0$. Values are normalized by the mean value of flux at $\delta=0$. (b) Magnitude of the flux at $\delta=3.5 \sigma_{0}$, showing the emergence of preferential flow paths in a highly stressed fracture. Values are normalized by the mean value of flux at $\delta=3.5 \sigma_{0}$. (c) $x$-component of the flux at $\delta=3.5 \sigma_{0}$, normalized by the mean $x$-directional flux. The presence of fracture contacts (green areas) induce preferential flow paths and negative velocities in the $x$-direction. (d) Probability density distributions of total volumetric flux at four different displacements: $\delta=0$ (black), $1.5 \sigma_{0}$ (blue), $2.5 \sigma_{0}$ (green), and $3.5 \sigma_{0}$ (red). The flux magnitude becomes significantly broader as stress increases. Inset: change in the fraction of contact area in the fracture as a function of displacement $\delta$. Symbols correspond to the four $\delta$ values above (colors matched). 
probability, and the transition time is $\tau_{i}=\frac{a_{i} l}{\frac{1}{2} \sum_{j}\left|q_{i j}\right|}$, where $a_{i}$ is the aperture value at grid block $i, l$ is the size of the grid block (assumed square), and $q_{i j}$ are the edge fluxes.

To initialize the streamlines, we inject $10^{4}$ particles at the inlet face with flux-weighted probability. To obtain particle trajectories that are long enough to observe macroscopic behavior, we concatenate particle trajectories randomly with flux-weighted probability (we have confirmed that the flux distribution at inlet and outlet faces are virtually identical).

The change in the transport behavior due to the increase in stress can be clearly observed from particle breakthrough curves and from the temporal evolution of the longitudinal mean square displacement (MSD) with respect to the center of mass. The peak arrival time is delayed, and particle breakthrough curves become heavy-tailed as $\delta$ increases [Figure 4(a)]. The late-time scaling of the longitudinal MSD is Fickian for low-stress fractures (MSD $\sim t^{1}$ for $\delta<2 \sigma_{0}$ ), and becomes strongly anomalous (superdiffusive) for highly stressed fractures $\left(\mathrm{MSD} \sim t^{\alpha}\right.$ with $\alpha>1$ for $\left.\delta>3 \sigma_{0}\right)$.

The complete mixing rule is a widely used method when solving transport through fractures (Dverstorp et al., 1992; Berkowitz et al., 1994; Stockman et al. 1997; Park et al., 2001), which assumes that Péclet numbers at grid blocks are small enough that particles are well mixed within the grid block. We have compared the results with simulations of particle transport using a streamline routing protocol, which assumes that Péclet numbers at grid blocks are large enough that particles follow the streamlines and do not transition between streamlines, and confirmed that the spreading behavior is insensitive to the mixing rule, in accord with earlier findings for transport in heterogeneous conductivity fields (Park et al., 2001; Kang et al., 2015a) (see Appendix A.1). While it is true that diffusion will eventually revert particle spreading to Fickian behavior (Dentz et al., 2004), the lack of sensitivity to the grid block mixing 175 rule indicates that diffusion does not appear to play any significant role even for the long simulation times we study (dimensionless times $t \sim 10^{4}$ ). However, a rigorous account of the effect of molecular diffusion at specific Péclet num- 

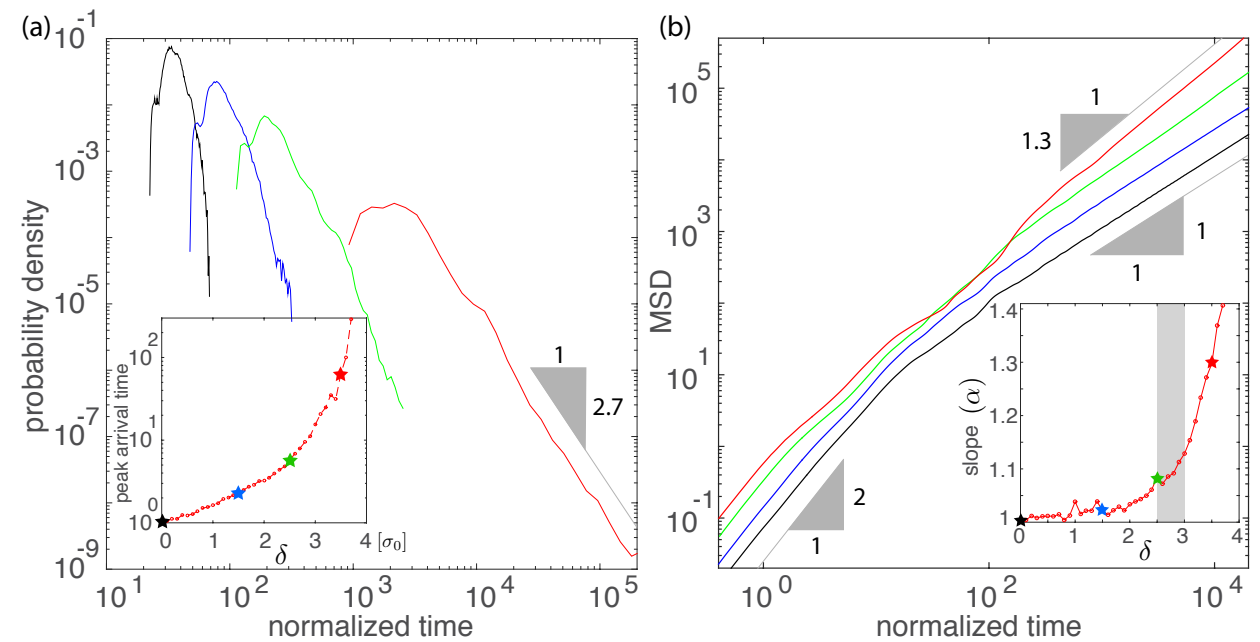

Figure 4: (a) Particle breakthrough curves measured at $x=L / 8$, for four different stress values corresponding to normal displacements $\delta=0$ (black), $1.5 \sigma_{0}$ (blue), $2.5 \sigma_{0}$ (green), and $3.5 \sigma_{0}$ (red). Inset: delay in peak arrival time as a function of $\delta$. (b) Temporal evolution of particle mean square displacement (MSD) for the four values of $\delta$. Time is normalized with the mean transition time over one grid block at the corresponding $\delta$. The transport behavior experiences a transition from normal (slope $=1$ ) to anomalous (slope $>1$, super-diffusive). Inset: evolution of late-time power-law scaling of MSD with time as a function of $\delta$. The transition from Fickian to non-Fickian transport occurs at $\delta \approx 2.5 \sigma_{0}$.

bers would require a particle tracking simulation with advective and diffusive steps (e.g., Bijeljic et al., 2011).

We have explored different values of parameters $D_{f}, k_{c}$ and $\theta$, and confirmed that the emergence of anomalous transport in stressed rough fractures is a general phenomenon, independently of the details of fracture roughness, correlation cut-off and decorrelation rate (see Appendix A.2 and Appendix A.3). We have also confirmed that the late-time spreading behavior obtained from one realization are representative of an ensemble of realizations at all levels of normal stress (see Appendix A.4). This is because the domain size is large enough to represent the statistics of a given fracture geometry, so that the results are also independent of domain size. 


\section{Origin of anomalous transport}

190 anomalous transport, we analyze Lagrangian velocity statistics - in particular, transition time distribution and velocity correlation.

The transition time distribution, $\psi_{\tau}(t)$, is the fundamental descriptor of the continuous time random walk (CTRW) formalism (Scher \& Montroll, 1975 quantify velocity correlation, we calculate the normalized velocity correlation time, $\tau_{c}=\int_{0}^{\infty} d t \chi_{v}(t / \bar{t})$ where $\chi_{v}(t)$ is the velocity autocorrelation function in time and $\bar{t}$ is the mean advective time over $\ell=\Delta x . \tau_{c}$ initially decreases as 
$\delta$ increases, exhibits a minimum around $\delta=2.5 \sigma_{0}$, and then increases again

${ }_{220}$ [Figure [5(a),inset 2]. We conjecture that the initial decrease in $\tau_{c}$ is due to the emergence of a more heterogeneous velocity field as the fracture is stressed. This leads to more heterogeneous particle velocity trajectory that induces a reduction in velocity correlation. However, for higher levels of stress, strong preferential paths and stagnation zones emerge [Figure 3(b)]; particles tend to remain in these preferential paths (or in the stagnation zones) with similar velocity magnitude, thereby increasing the velocity correlation. Interestingly, the level of stress at which $\tau_{c}$ is minimum coincides with that for the transition from normal to anomalous transport $\left(\delta \approx 2.5 \sigma_{0}\right)$.

\section{Spatial Markov model}

To develop an effective model for the average particle density, we extend a spatial Markov model (Kang et al., 2015b) and characterize velocity distribution and velocity correlation with three parameters: $\tau_{0}, \beta$ and $\xi$. Velocity distribution is captured by a transition time distribution that follows truncated power law [Figure 5 (a)]: $\psi_{\tau}\left(t ; \tau_{0}, \beta\right)=\frac{1}{\tau_{0}^{-\beta} \Gamma(\beta, 0)} \exp \left(-\frac{\tau_{0}}{t}\right) t^{-(1+\beta)}$, where $\tau_{0}$ determines the early time cutoff, $\beta$ the power-law slope, and $\Gamma(\beta, 0)=$ $\int_{0}^{\infty} t^{\beta-1} \exp (-t) d t$.

Recent studies of transport through porous media at various scales have shown that particle velocities sampled in space form a Markov process (Le Borgne et al., 2008; Kang et al., 2011b; de Anna et al., 2013; Kang et al., 2014).

In this case, the full velocity correlation information can be captured by the transition matrix (Le Borgne et al. 2008), where the transition times $\tau$ are discretized into $N$ classes such that the transition probabilities between classes are represented by the $N \times N$ transition matrix $\mathbf{T}$. While the full transition matrix obtained from direct numerical simulations can be complex, here we consider a simple transition-matrix model, in which all diagonal entries are fixed to a constant $A$, and the remaining entries are equal to $(1-A) /(N-1)$, that is, $T_{i j}=A \delta_{i j}+\frac{1-A}{N-1}\left(1-\delta_{i j}\right)$. This transition matrix imposes the same correlation 
function for all starting velocities, and the diagonal value of $A \leq 1$ determines the correlation strength. For this simple transition matrix, the correlation length $\xi$ is uniquely determined by the diagonal value $A$, and in the limit $N \gg 1$, $\xi=\ell / \ln \left(A^{-1}\right)$ (Kang et al. 2015b).

In short, $\beta$ determines the late-time scaling of MSD, $\tau_{0}$ determines the peak arrival time, and $\xi$ determines the transition time to anomalous scaling and thereby the magnitude of spreading. We estimate $\tau_{0}$ and $\beta$ from the transition time distribution and $\xi$ from the spatial velocity correlation function obtained from the direct numerical simulations, and use these parameter values for model prediction. The model exhibits excellent agreement with the direct simulations, and captures the transport behavior at all levels of stress, from normal to anomalous transport [Figure 5(b)].

\section{Summary}

We have shown that stress can induce anomalous transport in a rough fracture as a result of changes to the fundamental structure of the flow field, which organizes into preferential-flow channels and stagnation zones. We have proposed and validated a spatial Markov model that incorporates velocity distribution and correlation with only three physical parameters. While the results presented here are obtained for self-affine fractures, we have confirmed that the same transition behavior from normal to anomalous transport as a function of stress occurs also for other statistical descriptions of fracture roughness (see Appendix A.5, pointing to the universal character of our findings.

\section{Acknowledgments}

This work was funded by the US Department of Energy through a DOE CAREER Award (grant DE-SC0003907) and a DOE Mathematical Multifaceted Integrated Capability Center (grant DE-SC0009286). P.K.K. gratefully acknowledges support from the Korean Ministry of Land, Infrastructure and Transport 

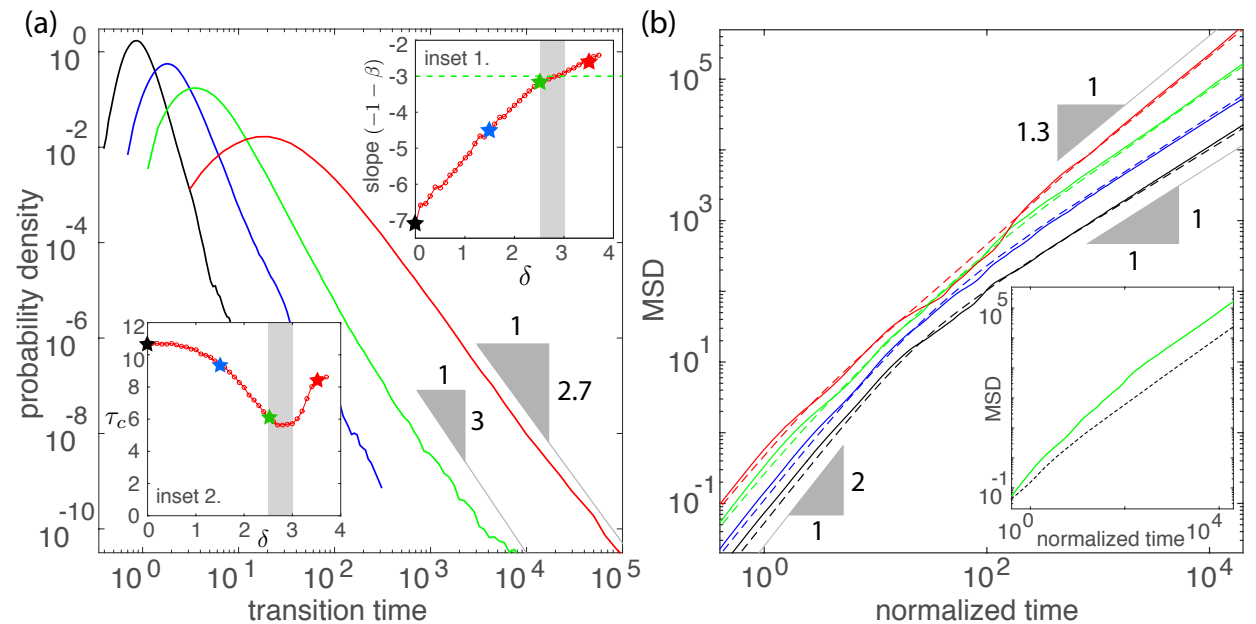

Figure 5: (a) Probability density distributions of Lagrangian transition time, $\psi_{\tau}(t)$, for four different normal displacements: $\delta=0$ (black), $1.5 \sigma_{0}$ (blue), $2.5 \sigma_{0}$ (green), and $3.5 \sigma_{0}$ (red). Inset 1: change in late-time scaling of $\psi_{\tau}(t)$ as a function of $\delta$. Anomalous transport is expected to occur when $\beta<2$, which occurs when $\delta>2.5 \sigma_{0}$. Inset 2 : change in the velocity correlation time $\tau_{c}$ as a function of $\delta$. The correlation time is minimum at the value of $\delta$ corresponding to the transition from normal to anomalous transport. (b) Time evolution of the MSDs from the direct simulation (solid lines) and the prediction with the spatial Markov model (dashed lines) for the four different values of $\delta$. Inset: Comparison between the MSD at $\delta=2.5 \sigma_{0}$ from direct simulation (green solid line) and the prediction with the CTRW model ignoring velocity correlation. Velocity correlation determines the transition time from ballistic to asymptotic scaling. 
275 (15AWMP-B066761-03). Data used in this manuscript can be obtained from the corresponding author (juanes@mit.edu).

\section{Appendix A.}

Appendix A.1. Results for different mixing rules

We do not explicitly consider diffusion in the simulations of transport. However, the complete mixing rule implicitly introduces diffusion at the scale of the grid block. The complete mixing rule is a widely used assumption when solving transport through fractures (Dverstorp et al., 1992 , Berkowitz et al., 1994 Stockman et al., 1997; Park et al., 2001), which assumes that Péclet numbers at grid blocks are small enough that particles are well mixed within the grid block.

${ }_{285}$ To assess whether our findings are independent of the assumption of full mixing at the computational grid blocks that define the fracture aperture field, we also simulate particle transport using a streamline routing protocol, which assumes that Péclet numbers at grid blocks are large enough that particles follow the streamlines and do not transition between streamlines. The complete mixing and streamline routing rules are two end members with regard to the importance of diffusion. Previous works have shown that longitudinal spreading is largely insensitive to the mixing rule in heterogeneous conductivity fields (Park et al. 2001, Kang et al. 2015a), and we have confirmed that this is also true for a rough fracture (Fig. A.6.

\section{Appendix A.2. Results for different levels of aperture fluctuations}

In Fig. A.7, we show simulation results for two different levels of aperture fluctuations $\left(D_{f}=2.3,2.6\right)$. We have confirmed that anomalous transport emerges as stress increases across all levels of fracture roughness and that, in this case, there was no noticeable difference due to the change in the roughness.

Appendix A.3. Results for different decay rates of roughness correlation

We show the emergence of anomalous transport for different correlation decay rates (Fig. A.8). While the late-time power-law scaling is indeed dependent 


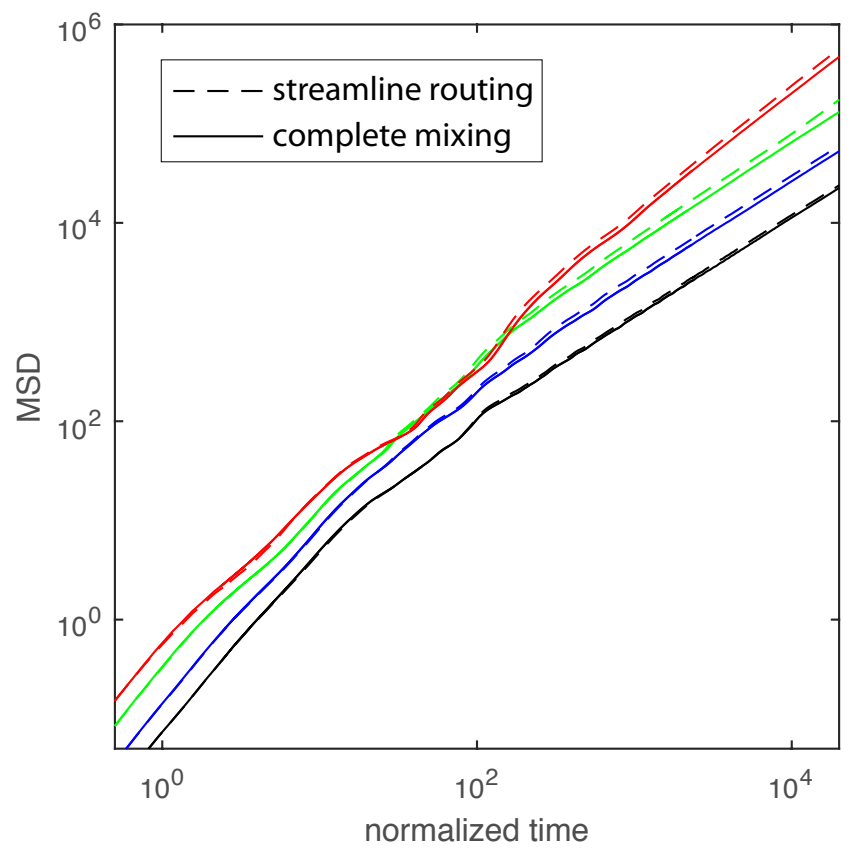

Figure A.6: Time evolution of MSD from the complete mixing rule (solid line) and streamline routing rule (dashed line) for four different values of $\delta$. The two cases show almost identical spreading behavior, and a transition from ballistic to super-diffusive regime. 
(a)
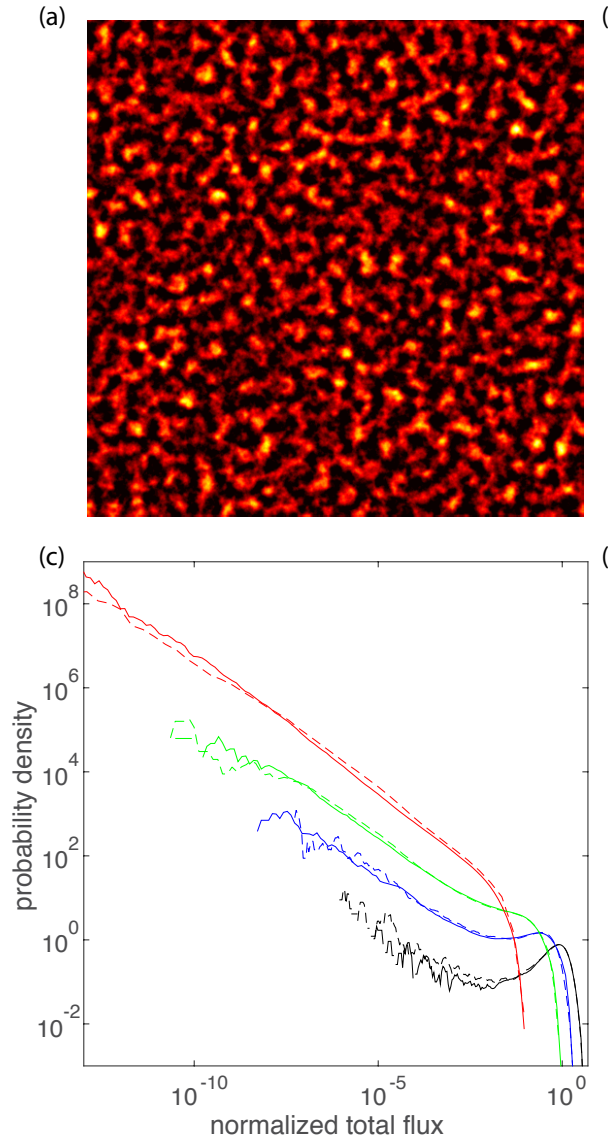

(b)

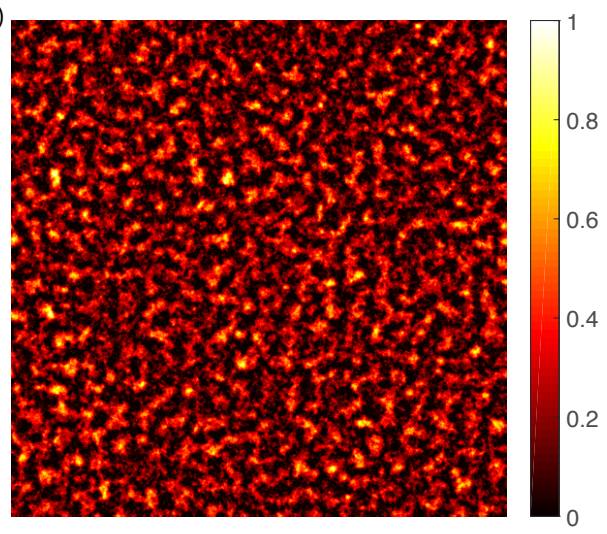

(d)

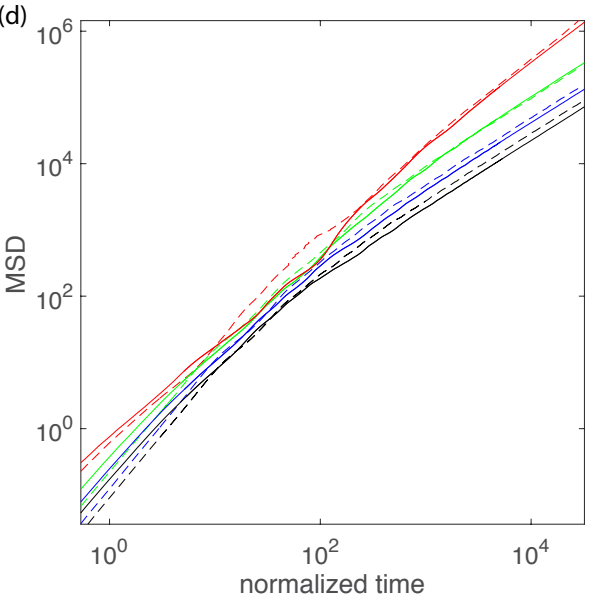

Figure A.7: $(\mathrm{a}, \mathrm{b})$ Aperture maps $a(x, y)$ between two rough surfaces with $D_{f}=2.3$ and $D_{f}=2.6$, respectively, at normal displacement $\eta=0.8$. (c) Probability density distributions of total volumetric flux at four different displacements $(\eta=0.8,1.8,2.6,3.5)$ for the two different values of fracture roughness (solid line for $D_{f}=2.6$, dashed line for $D_{f}=2.3$ ). There is no noticeable difference. (d) Time evolution of the MSDs for the four different displacements (solid line for $D_{f}=2.6$, dashed line for $D_{f}=2.3$ ). Both cases exhibit very similar flux distribution and late-time particle spreading behavior. 
on the decorrelation rate $\theta$, and the transition to anomalous transport occurs earlier for $\theta=8$ than for $\theta=4$, the general observation remains. Appendix A.4. Results for multiple realizations

We have confirmed that the results from one realization are representative by comparing simulation results for an ensemble of realizations. In Fig. A.9. we compare simulation results from 10 realizations. In this case, we choose as a reference point the mean aperture value at first contact, $\mu$ (Fig. A.9(a)). We define a normalized displacement with respect to the reference point, $\eta$, as follows: $\eta=\left(\delta^{\prime}-\mu\right) / \sigma$, where $\sigma$ is the standard deviation of aperture values at first contact (zero normal stress). We show that the aperture statistics, fluid flow statistics and long-time particle spreading behavior are virtually identical between realizations. This is because the domain size is large enough to represent the statistics of a given fracture geometry. Therefore, results from a single fracture lead to the same conclusions as results from ensemble averaging.

\section{Appendix A.5. Results for different models of fracture roughness}

Finally, we have confirmed the emergence of anomalous transport for different models of fracture roughness, which are not self-affine. To illustrate this, we present results of flow and transport in a Gaussian log-aperture field (Ruan \& McLaughlin, 1998) (Fig. A.10). 
(a)

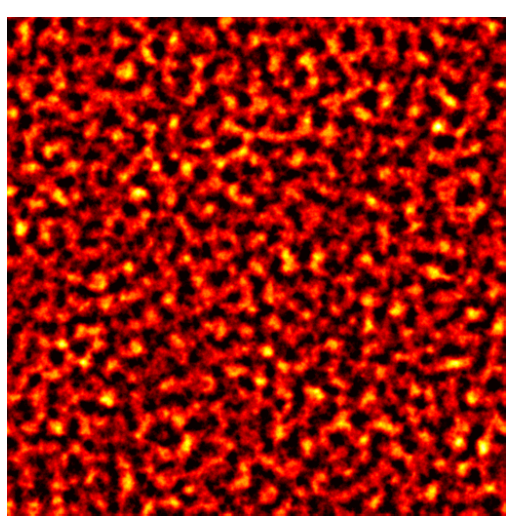

(c)

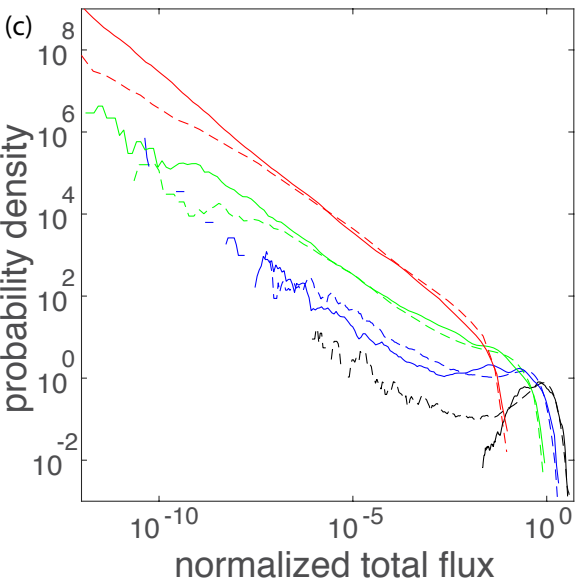

(b)

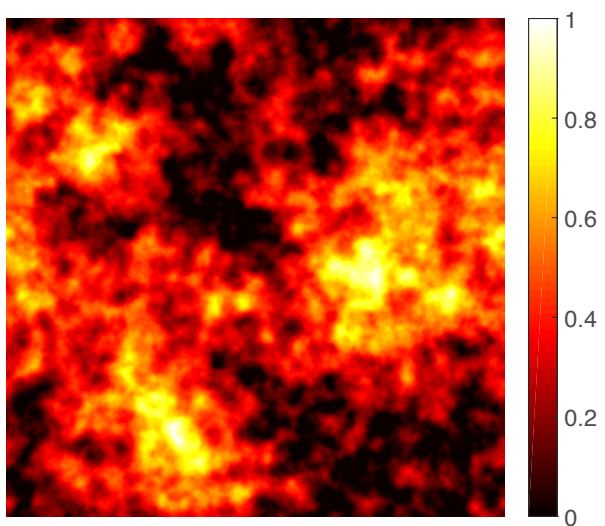

(d)

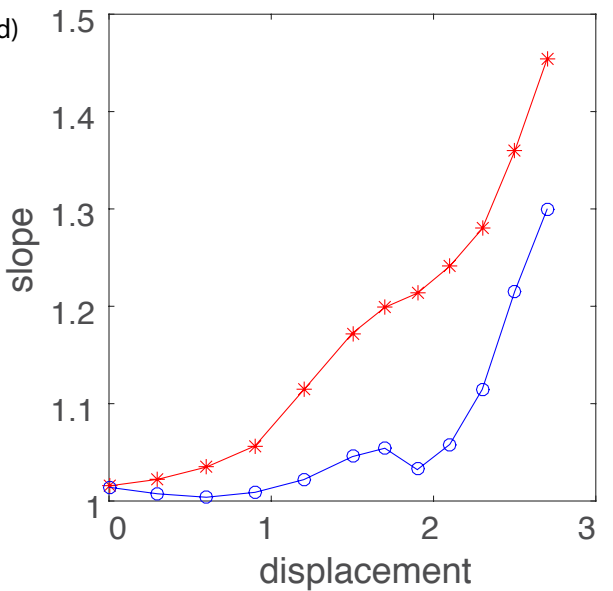

Figure A.8: $(\mathrm{a}, \mathrm{b})$ Aperture maps $a(x, y)$ between two rough surfaces with decorrelation rates $\theta=4$ and $\theta=8$, respectively, at normal displacement $\eta=0.8$. (c) Probability density distributions of total volumetric flux at four different normal displacements $(\eta=0.8,1.8,2.6,3.5)$ for the two different decorrelation rates (dashed line for $\theta=4$, solid line for $\theta=8$ ). (d) Evolution of late-time power-law scaling of MSD with time as a function of displacement. Displacement is defined as $|\eta-3.5|$. The transition to anomalous transport (slope $\alpha>1$ ) occurs earlier for $\theta=8$ (red stars) than for $\theta=4$ (blue circles). 
(a)

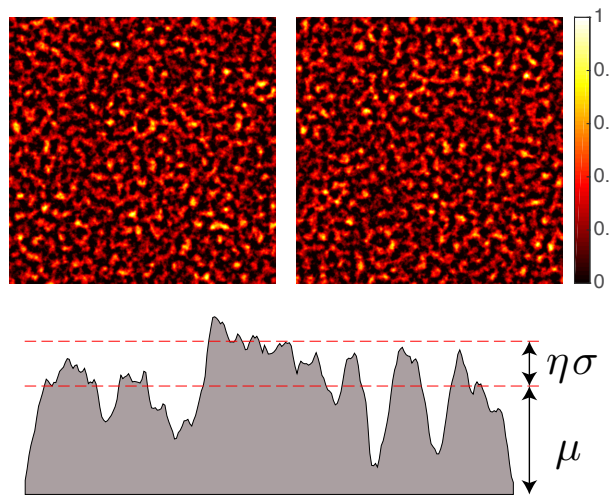

(c)

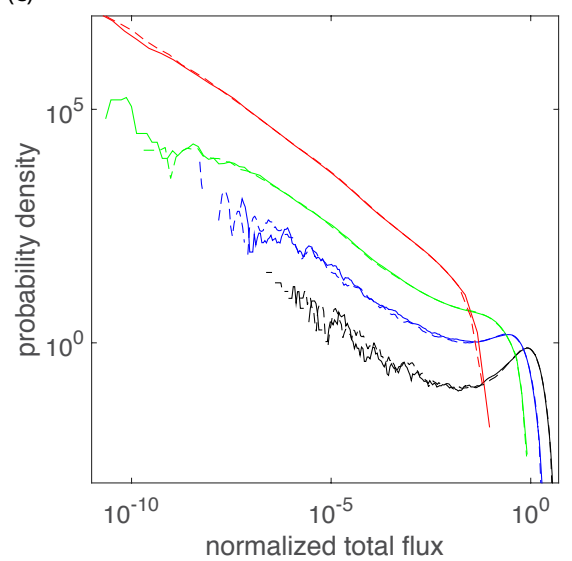

(b)

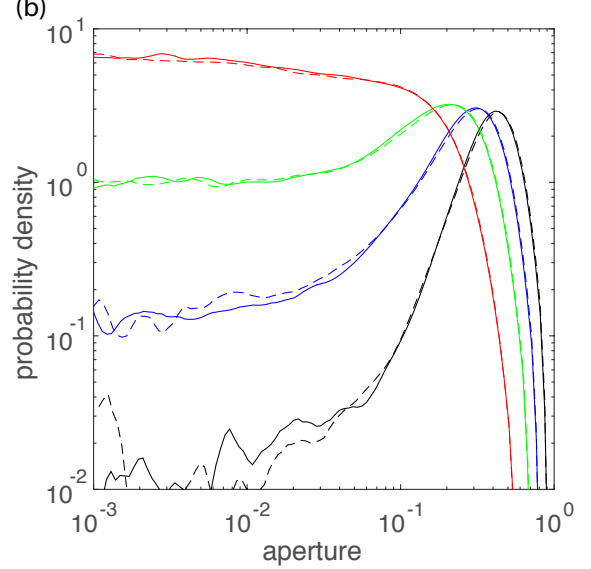

(d)

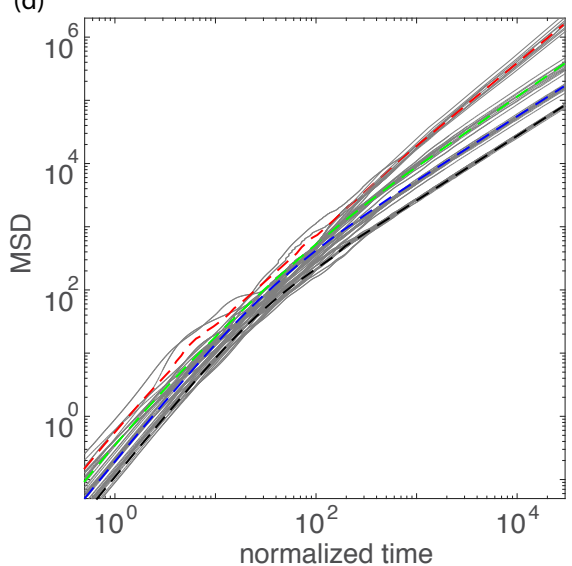

Figure A.9: (a) Two realizations of the fracture aperture field $a(x, y)$, at $\eta=0.8$. The schematic illustrates the reference point for the vertical displacements. (b) Probability density distributions of aperture at four different displacements $(\eta=0.8,1.8,2.6,3.5)$ for the two different realizations (solid line and dashed line for each realization). (c) Probability density distributions of total volumetric flux at four different displacements. (d) Time evolution of the MSDs for the four different displacements. We have run simulations over ten different realizations (gray lines) and the comparison with the average behavior (dashed line) shows that late-time spreading behavior obtained from one realization are representative of an ensemble of realizations at all levels of normal stress. The different realizations show virtually identical aperture distributions, flux distributions, and late-time particle spreading behavior, at all levels of normal stress. 
(a)

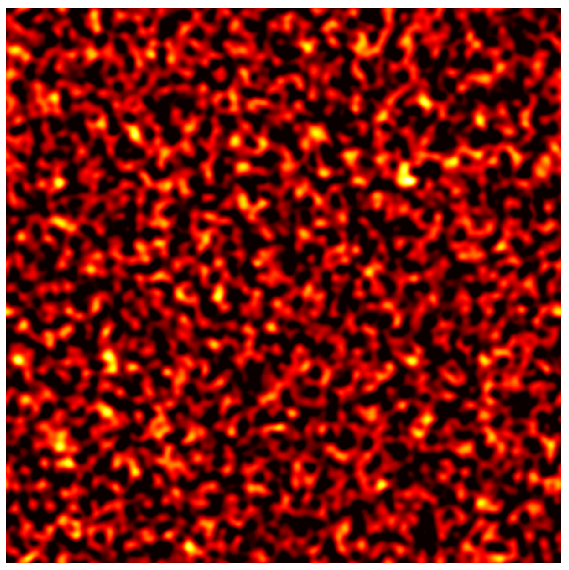

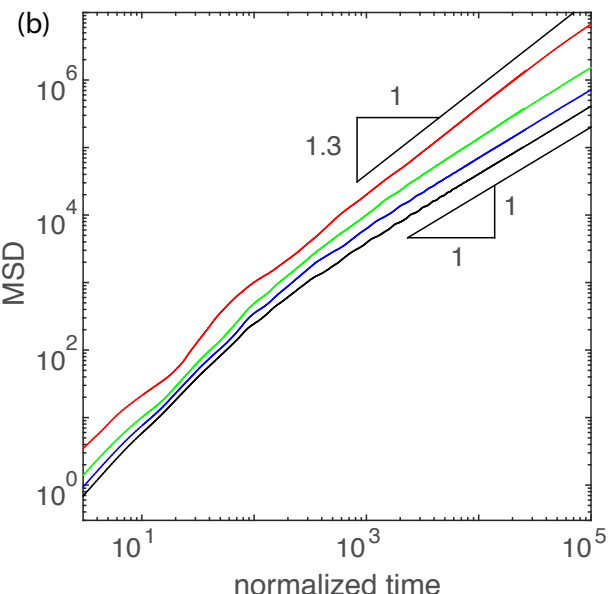

Figure A.10: (a) An aperture map $a(x, y)$ that follows a Gaussian log-aperture field, at normal displacement $\eta=0.8$. (b) Time evolution of the MSDs for four different values of the normal displacement. This Gaussian log-aperture field also leads to the emergence of anomalous transport in response to increasing confining stress. 


\section{References}

Andrews, D. J. (1988). On modeling closure of rough surfaces in contact. Eos, Trans. Am. Geophys. Union, 69, 1426.

325 de Anna, P., Le Borgne, T., Dentz, M., Tartakovsky, A. M., Bolster, D., \&

Davy, P. (2013). Flow intermittency, dispersion, and correlated continuous time random walks in porous media. Phys. Rev. Lett., 110, 184502.

Auradou, H. (2009). Influence of wall roughness on the geometrical, mechanical and transport properties of single fractures. J. Phys. D: Appl. Phys., 42, 214015.

Auradou, H., Hulin, J. P., \& Roux, S. (2001). Experimental study of miscible displacement fronts in rough self-affine fractures. Phys. Rev. E, 63, 066306.

Bandis, S. C., Lumsden, A. C., \& Barton, N. R. (1983). Fundamentals of rock joint deformation. Int. J. Rock Mech. Min. Sci. \& Geomech. Abstr., 20, 249-268.

Bear, J., Tsang, C. F., \& de Marsily, G. (1993). Flow and Contaminant Transport in Fractured Rock. Academic Press.

Becker, M. W., \& Shapiro, A. M. (2000). Tracer transport in fractured crystalline rock: Evidence of nondiffusive breakthrough tailing. Water Resour. Res., 36, 1677-1686.

Berkowitz, B., Cortis, A., Dentz, M., \& Scher, H. (2006). Modeling non-Fickian transport in geological formations as a continuous time random walk. Rev. Geophys., 44, RG2003.

Berkowitz, B., Naumann, C., \& Smith, L. (1994). Mass transfer at fracture intersections: An evaluation of mixing models. Water Resour. Res., 30, 17651773.

Berkowitz, B., \& Scher, H. (1997). Anomalous transport in random fracture networks. Phys. Rev. Lett., 79, 4038-4041. 
Bijeljic, B., Mostaghimi, P., \& Blunt, M. J. (2011). Signature of non-Fickian solute transport in complex heterogeneous porous media. Phys. Rev. Lett., $107,204502$.

Bodin, J., Delay, F., \& de Marsily, G. (2003a). Solute transport in a single fracture with negligible matrix permeability: 1. Fundamental mechanisms. Hydrogeol. J., 11, 418-433.

Bodin, J., Delay, F., \& de Marsily, G. (2003b). Solute transport in a single fracture with negligible matrix permeability: 2. Mathematical formalism. Hydrogeol. J., 11, 434-454.

Bodvarsson, G. S., B., W., Patterson, R., \& Williams, D. (1999). Overview of scientific investigations at Yucca Mountain: the potential repository for high-level nuclear waste. J. Contaminant Hydrol., 38, 3-24.

Bouchaud, J. P., \& Georges, A. (1990). Anomalous diffusion in disordered media - statistical mechanisms, models and physical applications. Phys. Rep., 195, $127-293$.

Brown, S. (1995). Simple mathematical model of a rough fracture. J. Geophys. Res., 100, 5941-5952.

Brown, S. R., \& Scholz, C. H. (1985). Closure of random elastic surfaces in contact. J. Geophys. Res., 90, 5531-5545.

Carrera, J., Sánchez-Vila, X., Benet, I., Medina, A., Galarza, G. A., \& Guimerá, J. (1998). On matrix diffusion: formulations, solution methods and qualitative effects. Hydrogeol. J., 6, 178-190.

Dentz, M., \& Bolster, D. (2010). Distribution- versus correlation-induced anomalous transport in quenched random velocity fields. Phys. Rev. Lett., $105,244301$.

Dentz, M., Cortis, A., Scher, H., \& Berkowitz, B. (2004). Time behavior of 375 solute transport in heterogeneous media: transition from anomalous to normal transport. Adv. Water Resour., 27, 155-173. 
Detwiler, R. L., Rajaram, H., \& Glass, R. J. (2000). Solute transport in variableaperture fractures: An investigation of the relative importance of Taylor dispersion and macrodispersion. Water Resour. Res., 36, 1611-1625.

Drazer, G., Auradou, H., Koplik, J., \& Hulin, J. P. (2004). Self-affine fronts in self-affine fractures: Large and small-scale structure. Phys. Rev. Lett., 92, 014501.

Dverstorp, B., Andersson, J., \& Nordqvist, W. (1992). Discrete fracture network interpretation of field tracer migration in sparsely fractured rock. Water Resour. Res., 28, 2327-2343.

Geiger, S., Cortis, A., \& Birkholzer, J. T. (2010). Upscaling solute transport in naturally fractured porous media with the continuous time random walk method. Water Resour. Res., 46, W12530.

Glover, P. W. J., Matsuki, K., Hikima, R., \& Hayashi, K. (1998). Synthetic rough fractures in rocks. J. Geophys. Res., 103, 9609-9620.

Hopkins, D. L. (1990). The Effect of Surface Roughness on Joint Stiffness, Aperture, and Acoustic Wave Propagation. PhD Dissertation.

Ishibashi, T., Watanabe, N., Hirano, N., Okamoto, A., \& Tsuchiya, N. (2015). Beyond-laboratory-scale prediction for channeling flows through subsurface rock fractures with heterogeneous aperture distributions revealed by laboratory evaluation. J. Geophys. Res., 120, 106-124.

Jing, L., Koyama, T., Zhao, Z., \& Li, B. (2013). Stress and shear effects on fluid flow and solute transport in rock fractures. In 3rd ISRM Symposium on Rock Characterisation, Modelling and Engineering Design Methods, SINOROCK 2013; Shanghai, China (pp. 33-44). Taylor \& Francis Group.

Johnson, R. B., \& DeGraff, J. V. (1988). Principles of Engineering Geology. Wiley. 
Kang, P. K., de Anna, P., Nunes, J. P., Bijeljic, B., Blunt, M. J., \& Juanes, R. (2014). Pore-scale intermittent velocity structure underpinning anomalous transport through 3-d porous media. Geophys. Res. Lett., 41, 6184-6190, doi:10.1002/2014GL061475.

Kang, P. K., Dentz, M., \& Juanes, R. (2011a). Predictability of anomalous transport on lattice networks with quenched disorder. Phys. Rev. E, 83, 030101(R), doi:10.1103/PhysRevE.83.030101.

Kang, P. K., Dentz, M., Le Borgne, T., \& Juanes, R. (2011b). Spatial Markov model of anomalous transport through random lattice networks. Phys. Rev. Lett., 107, 180602, doi:10.1103/PhysRevLett.107.180602.

Kang, P. K., Dentz, M., Le Borgne, T., \& Juanes, R. (2015a). Anomalous transport on regular fracture networks: Impact of conductivity heterogeneity and mixing at fracture intersections. Phys. Rev. E, 92, 022148.

Kang, P. K., Le Borgne, T., Dentz, M., Bour, O., \& Juanes, R. (2015b). Impact of velocity correlation and distribution on transport in fractured media: field evidence and theoretical model. Water Resour. Res., 51, 940-959, doi:10.1002/2014WR015799.

${ }_{420}$ Klafter, J., \& Silbey, R. (1980). Derivation of the continuous-time random-walk equation. Phys. Rev. Lett., 44, 55-58.

Koyama, T., Li, B., Jiang, Y., \& Jing, L. (2008). Numerical simulations for the effects of normal loading on particle transport in rock fractures during shear. Int. J. Rock Mech. Min. Sci., 45, 1403-1419.

Le Borgne, T., Dentz, M., \& Carrera, J. (2008). Lagrangian statistical model for transport in highly heterogeneous velocity fields. Phys. Rev. Lett., 101, 090601.

Måløy, K. J., Feder, J., Boger, F., \& Jøssang, T. (1988). Fractal structure of hydrodynamic dispersion in porous media. Phys. Rev. Lett., 61, 2925-2928. 
Metzler, R., \& Klafter, J. (2000). The random walks guide to anomalous diffusion: a fractional dynamics approach. Phys. Rep., 339, 1-77.

Meyer, D. W., \& Tchelepi, H. A. (2010). Particle-based transport model with Markovian velocity processes for tracer dispersion in highly heterogeneous porous media. Water Resour. Res., 46, W11552.

Moreno, L., \& Neretnieks, I. (1993). Fluid flow and solute transport in a network of channels. J. Contaminant Hydrol., 14, 163-194.

Moreno, L., Tsang, Y. W., Tsang, C. F., Hale, F. V., \& Neretnieks, I. (1988). Flow and tracer transport in a single fracture: A stochastic model and its relation to some field observations. Water Resour. Res., 24, 2033-2048.

${ }_{440}$ Nemoto, K., Watanabe, N., Hirano, N., \& Tsuchiya, N. (2009). Direct measurement of contact area and stress dependence of anisotropic flow through rock fracture with heterogeneous aperture distribution. Earth Planet. Sci. Lett., $281,81-87$.

Olsson, W. A., \& Brown, S. R. (1993). Hydromechanical response of a fracture undergoing compression and shear. Int. J. Rock Mech. Min. Sci., 30, 845-851.

Park, Y. J., de Dreuzy, J. R., Lee, K. K., \& Berkowitz, B. (2001). Transport and intersection mixing in random fracture networks with power law length distributions. Water Resour. Res., 37, 2493-2501.

Petrovitch, C. L., Pyrak-Nolte, L. J., \& Nolte, D. D. (2014). Combined scaling of fluid flow and seismic stiffness in single fractures. Rock Mech. Rock Eng., $47,1613-1623$.

Pollard, D. D., \& Fletcher, R. C. (2005). Fundamentals of Structural Geology. Cambridge University Press.

Power, W. L., \& Tullis, T. E. (1991). Euclidean and fractal models for the 455 description of rock surface roughness. J. Geophys. Res., 96, 415-424. 
Pruess, K. (2006). Enhanced geothermal systems (EGS) using $\mathrm{CO}_{2}$ as working fluid: novel approach for generating renewable energy with simultaneous sequestration of carbon. Geothermics, 35, 351-367.

Pyrak-Nolte, L. J., \& Morris, J. P. (2000). Single fractures under normal stress: The relation between fracture specific stiffness and fluid flow. Int. J. Rock Mech. Min. Sci., 37, 245-262.

Pyrak-Nolte, L. J., \& Nolte, D. D. (2016). Approaching a universal scaling relationship between fracture stiffness and fluid flow. Nature Comm., 7, 10663.

Ruan, F., \& McLaughlin, D. (1998). An efficient multivariate random field generator using the fast Fourier transform. Adv. Water Resour., 21, 385399.

Scher, H., \& Montroll, E. W. (1975). Anomalous transit-time dispersion in amorphous solids. Phys. Rev. B, 12, 2455-2477.

Seymour, J. D., Gage, J. P., Codd, S. L., \& Gerlach, R. (2004). Anomalous fluid transport in porous media induced by biofilm growth. Phys. Rev. Lett., 93, 198103.

Shlesinger, M. F. (1974). Asymptotic solutions of continuous-time random walks. J. Stat. Phys., 10, 421-434.

Stockman, H. W., Li, C., \& Wilson, J. L. (1997). A lattice-gas and lattice Boltzmann study of mixing at continuous fracture junctions: Importance of boundary conditions. Geophys. Res. Lett., 24, 1515-1518.

Talon, L., Auradou, H., \& Hansen, A. (2012). Relation between first arrival time and permeability in self-affine fractures with areas in contact. Europhys. Lett., 97, 68009.

${ }_{480}$ Tsang, Y. W., \& Tsang, C. F. (1987). Channel model of flow through fractured media. Water Resour. Res., 23, 467-479. 
Unger, A. J. A., \& Mase, C. W. (1993). Numerical study of the hydromechanical behavior of two rough fracture surfaces in contact. Water Resour. Res., 29, 2101-2114.

Vilarrasa, V., Koyama, T., Neretnieks, I., \& Jing, L. (2011). Shear-induced flow channels in a single rock fracture and their effect on solute transport. Transp. Porous Media, 87, 503-523.

Wang, H. F. (2000). Theory of Linear Poroelasticity. Princeton University Press.

Wang, L., \& Cardenas, M. B. (2014). Non-Fickian transport through twodimensional rough fractures: Assessment and prediction. Water Resour. Res., $50,871-884$.

Watanabe, N., Hirano, N., \& Tsuchiya, N. (2008). Determination of aperture structure and fluid flow in a rock fracture by high-resolution numerical modeling on the basis of a flow-through experiment under confining pressure. Water Resour. Res., 44, W06412.

Watanabe, N., Ishibashi, T., Tsuchiya, N., Ohsaki, Y., Tamagawa, T., Tsuchiya, Y., Okabe, H., \& Ito, H. (2013). Geologic core holder with a CFR PEEK body for the X-ray CT-based numerical analysis of fracture flow under confining pressure. Rock Mech. Rock Eng., 46, 413-418.

Yasuhara, H., Polak, A., Mitani, Y., Grader, A. S., Halleck, P. M., \& Elsworth, D. (2006). Evolution of fracture permeability through fluidrock reaction under hydrothermal conditions. Earth Planet. Sci. Lett., 244, 186-200. 